\title{
Magnetic resonance neuroimaging promotes the preservation of pelvic autonomic nerves in laparoscopic total mesorectal excision: a comparative study
}

\author{
Guangyu Zhong ${ }^{1 \#}$, Bin Yang ${ }^{1 \#}$, Jinglian Zhong ${ }^{2 \#}$, Yingkui Zhong ${ }^{3}$, Shilin Zhi ${ }^{1}$, Jun Shen ${ }^{2}$, \\ Shengning Zhou ${ }^{1}$, Jia'nan Tan ${ }^{1}$, Jing Huang ${ }^{4}$, Jiankun Zhu' ${ }^{1}$, Dongye Wang ${ }^{2}$, Fanghai Han ${ }^{1}$ \\ ${ }^{1}$ Department of Gastrointestinal Surgery, Sun Yat-sen Memorial Hospital, Sun Yat-sen University, Guangzhou, China; ${ }^{2}$ Department of Radiology, \\ Sun Yat-sen Memorial Hospital, Sun Yat-sen University, Guangzhou, China; ${ }^{3}$ Department of Gastroenterology, the Sixth Affiliated Hospital, Sun \\ Yat-sen University, Guangzhou, China; ${ }^{4}$ Thyroid and Parathyroid Surgery Center, West China Hospital of Sichuan University, Chengdu, China \\ Contributions: (I) Conception and design: G Zhong, B Yang, J Zhong; (II) Administrative support: J Shen, F Han, D Wang; (III) Provision of \\ study materials or patients: G Zhong, B Yang, S Zhou; (IV) Collection and assembly of data: S Zhou, J Tan, J Huang, J Zhu; (V) Data analysis and \\ interpretation: G Zhong, Y Zhong, S Zhi; (VI) Manuscript writing: All authors; (VII) Final approval of manuscript: All authors. \\ \#These authors contributed equally to this work. \\ Correspondence to: Fanghai Han; Dongye Wang. Sun Yat-sen Memorial Hospital, Sun Yat-sen University, Yanjiang West Road No. 107, Yuexiu \\ District, Guangzhou 510120, China. Email: fh_han@163.com; wangdye@mail.sysu.edu.cn.
}

Background: How to preserve pelvic autonomic nerves system (PANS) in total mesorectal excision (TME) is still a technical challenge for gastrointestinal surgeons, and nerve preservation according to preoperative magnetic resonance imaging (MRI) is a hot topic in pelvic surgery. The purpose of this study was to assess the postoperative urogenital function of patients with rectal cancer (RC) who underwent preoperative and postoperative neuroimaging of PANS vs. patients who did not.

Methods: Patients meeting the inclusion criteria were prospectively enrolled in a magnetic resonance neuroimaging (MRN) group from June 2018, while primary RC patients from January 2016 to May 2018 who met the inclusion criteria were enrolled in a non-MRN group. Patients in the MRN group underwent MRN examination before operation and 6 months after operation, while those in the non-MRN group were collected and analyzed retrospectively.

Results: Based on International Prostate Symptom Score (IPSS) and International Index of Erectile Function 5 (IIEF5) scores at 6 months, the postoperative urinary and sexual function of male patients in the MRN group were significantly better than that in the non-MRN group $(\mathrm{P}<0.05)$. In addition, based on International Consultation on Incontinence modular Questionnaire on Female Lower Urinary Tract Symptoms (ICIQ-FLUTS) and Female Sexual Function Index (FSFI) scores at 6 months, the postoperative sexual function of female patients in the MRN group was significantly better than that in the non-MRN group $(\mathrm{P}<0.05)$.

Conclusions: In the present study, we constructed a three-dimensional (3D) presentation of PANS based on preoperative MRN which showed in vivo pelvic autonomous innervation. This may promote the preservation of PANS during TME and reduce the postoperative urogenital dysfunction rate.

Keywords: Rectal cancer (RC); magnetic resonance neuroimaging (MRN); pelvic autonomic nerve; prevalence of visibility (POV); urogenital function

Submitted Sep 22, 2021. Accepted for publication Dec 08, 2021.

doi: $10.21037 /$ atm-21-5658

View this article at: https://dx.doi.org/10.21037/atm-21-5658 


\section{Introduction}

Guidelines for the treatment of rectal cancer (RC) are well established (1-3), and the postoperative overall 5-year survival rate can be up to $70 \%(4,5)$. For most RC patients diagnosed with stage T1-3, total mesorectal excision (TME) surgery is the preferred treatment. However, complications can affect the long-term quality of life of patients after total mesorectal excision (TME) for RC including nerve injury-related injuries causing urinary and sexual dysfunction $(6,7)$. Threedimensional (3D) laparoscopic and Da Vinci robotic systems are characterized by high resolution and three-dimensional presentation. The magnified vision of 3D laparoscopic and Da Vinci robotic systems allows surgeons a better view for separation along fascia gaps, the postoperative urinary and sexual dysfunction rate of the $\mathrm{Da}$ Vinci robotic system is still high, with rates reported from $20-30 \%$ and $0-36 \%$ (8-10). The main reason for pelvic autonomic nerves system (PANS) injury is the lack of preoperative assessment, so gastrointestinal surgeons perform TME and autonomic nerve preservation (TME + ANP) according to their own experience and autopsy. However, how to avoid injury to PANS in TME remains a technical challenge for surgeons, and nerve preservation according to preoperative imaging has become a hot topic in pelvic surgery (11). Magnetic resonance neuroimaging (MRN) is one of the sequences of magnetic resonance imaging (MRI), which selectively enhances neural signals so that nerve can be showed clearly on MRN images. Currently, MRN of PANS is mainly based on anatomical studies of fetal and adult cadavers. In 2014, MRN was conducted on eight adult cadavers and showed a 3D model based on MRN was suitable to show the course of PANS (12), and in 2018, Li et al. (13) fully demonstrated the course of PANS and their navigation role in obstetrics and gynecology through MRN. However, there are no reports examining preoperative $\mathrm{MRN}$ in RC patients. In this study we performed MRN preoperatively in RC patients to demonstrate the course and distribution of PANS to evaluate if preoperative MRN images could promote preservation of PANS in laparoscopic TME + ANP surgery. We present the following article in accordance with the STROBE reporting checklist (available at https://dx.doi.org/10.21037/atm-215658).

\section{Methods}

\section{Patients}

All procedures performed in this study involving human participants were in accordance with the Declaration of Helsinki (as revised in 2013). This study was approved by the clinical ethics committee of Sun Yat-sen Memorial Hospital, Sun Yat-sen University (No. SYSEC-KYKS-2021-247) and informed consent was taken from all the patients.

Inclusion criteria: (I) primary $\mathrm{RC}$ diagnosed as an adenocarcinoma by electronic colonoscope and pathologic biopsy; (II) the lower margin of the tumor was within $12 \mathrm{~cm}$ from the anal verge; (III) aged 18-70 years; (IV) In males, the International Index of Erectile Function 5 (IIEF5) scored 22-25 and the International Prostate Symptom Score (IPSS) scored 0-7 preoperatively; In females, the Female Sexual Function Index (FSFI) scored 25-36 preoperatively; (V) clinical stage was T1-4aN0-2M0; (VI) circumference marge (CRM) negative evaluated by MRI.

Exclusion criteria: (I) multivisceral resection; (II) abdominoperineal resection; (III) lateral dissection of pelvic lymph nodes; (IV) conversion of laparoscopic surgery to open surgery; (V) multiple colorectal cancers (CRCs); (VI) number of harvested lymph nodes $<12$; (VII) central or peripheral nervous system lesions; (VIII) postoperative anastomotic leakage, pelvic abscess, or local recurrence.

MRN group: from June 2018 to December 2018, patients with primary RC admitted to Sun Yat-sen Memorial Hospital of Sun Yat-sen University were included and signed informed consent. IPSS, IIEF5 or FSFI, and International Consultation on Incontinence modular Questionnaire on Female Lower Urinary Tract Symptoms (ICIQ-FLUTS) were then filled out and MR and MRN performed. Patients who met the inclusion criteria were finally engaged in the MRN group. Urogenital function was reassessed by the forms mentioned above before operation if the patient was treated with neoadjuvant chemoradiotherapy.

According to the National Comprehensive Cancer Network (NCCN) standard (14), a long course of neoadjuvant chemoradiotherapy [radiotherapy (50.4 Gy) plus capecitabine $\left(1,000 \mathrm{mg} / \mathrm{m}^{2}\right.$, oral, twice a day, day $1-14$, day 22-35) for 5 weeks] was performed for patients with clinical $\mathrm{T} 3+$ and $\mathrm{N}+$ stages, and the reevaluation completed at 6-8 weeks. Patients were included if CRM reevaluated by MRI was negative.

Non-MRN group: data were collected and analyzed retrospectively, including patients with primary $\mathrm{RC}$ admitted to the Gastrointestinal Surgery Department of Sun Yat-sen Memorial Hospital from January 2016 to May 2018. Their urogenital functions before and 6 months 
Table 1 Characteristic of MRN sequence

\begin{tabular}{lc}
\hline Sequence parameter & 3D-NerveVIEW \\
\hline TR $(\mathrm{msec})$ & 2,200 \\
TE $(\mathrm{msec})$ & 170 \\
Field-of-view $(\mathrm{mm} \times \mathrm{mm})$ & $250 \times 250$ \\
Slice thickness $(\mathrm{mm})$ & 2 \\
Intersection gap & -1 \\
Number of slices & 130 \\
Matrix $(\mathrm{mm} \times \mathrm{mm})$ & $256 \times 256$ \\
\hline
\end{tabular}

TR, repetition time; TE, echo time; MRN, magnetic resonance neuroimaging.

after operation based on IPSS, IIEF5 or FSFI, and ICIQFLUTS were compared with that of the MRN group.

\section{MRN imaging sequence and parameter}

3D-NerveVIEW was performed and rebuilt by a Philips Ingenia DNA II 3.0 T MRI scanner with abdominal phased-array coil of multi-channel. Patients were not medicated to inhibit intestinal motility prior to imaging, and our scanning angle correspondingly changed according to different tilt angles of the sacral coccygeal vertebra. A 3D model based on Two-dimensional (2D) segmentation was then created using the $3 \mathrm{D}$ calculation function of ITK SNAP. The procedure took approximately 40 minutes to complete the scan and one hour to build a 3D model. All patients in the MRN group underwent MRN examination before operation and 6 months after operation, and the parameters are shown in Table 1.

\section{Inter-observer agreement}

The level of agreement was determined by gastrointestinal surgeons and radiologists specializing in pelvic anatomy and using Likert scales $(0=$ disagree that the depicted nerve depicted the correct nerve, $1=\mathrm{I}$ suspect the depicted nerve depicted the correct nerve, and 2= I agree that the depicted nerve depicted the correct nerve). We defined scores of 2 as successful visualization of PANS, and the success rate measure was defined as the number of successfully visualized nerves divided by the total number of nerves. The level of inter-observer agreement was then calculated.

\section{$T M E+A N P$ for patients with $R C$}

Before surgery, surgeons reviewed the MRI and MRN images again to confirm the course and distribution of PANS, the location of the tumor, and TNM stage. All operations were performed by the same surgical team, who had performed more than 100 laparoscopic TME + ANP every year through 3D laparoscopy (Braun).

The following steps for a standardized approach in TME + ANP were considered: Standard position and trocar placement; exposure; a tailored separation approach along the fascia gap between the prehypogastric nerve fascia (PHGNF) and rectal propria fascia according to preoperative MRN; vascular dissection and ligation; and radical resection for $\mathrm{RC}$. There are three vulnerable sites in laparoscopy TME + ANP:

(I) Hypogastric nerve (HGN): due to the variation of its emitting position and course, it is necessary to perform preoperative MRN to assist in confirming the course of the HGN, which involves separation between the rectal propria fascia and PHGNF.

(II) Pelvic splanchnic nerve (PSN): while the PSN is generally thought to be composed of the second sacral nerve (S2), the third sacral nerve (S3), and the fourth sacral nerve (S4), anatomy studies have revealed it could also originate from the first sacral nerve (S1). As the PSN joins the pelvic plexus without obvious pattern, its origin should be confirmed based on preoperative MRN.

(III) Pelvic plexus (PP): this should be separated along the posterior and anterolateral loose spaces between the white PHGNF and the yellow rectal propria fascia.

\section{Intraoperative evaluation}

MRN group: the superior hypogastric plexus (SHP), HGN, PSN, PP, neurovascular bundle (NVB), and sympathetic trunk at the root of inferior mesenteric artery (IMA) were observed and recorded intraoperatively.

Non-MRN group: the prevalence of visibility (POV) was calculated by reviewing patients' surgical videos and pictures.

The POV of nerves mentioned above in the MRN group were compared with that of the non-MRN group, and univariate and multivariate analyses were performed for the 
different results between the two.

\section{Postoperative evaluation}

MRN was performed 6 months after TME in the MRN group, and the postoperative result compared with the preoperative result. In addition, IPSS and IIEF5 were evaluated for male patients, and ICIQ-FLUTS and FSFI were evaluated for female patients, which were compared with preoperative results, respectively.

\section{Statistical analyses}

Statistical analyses were carried out using R x64 3.6.1. The kappa concordance test was used to analyze interobserver agreement and the chi-square or Fisher's exact test were used for categorical variables. Logistic regression was used for univariate analysis and multi-factor analysis, and variables of which $\mathrm{P}<0.10$ in univariate analysis were brought into multi-factor analysis. $\mathrm{P}<0.05$ was considered statistically significant.

\section{Results}

\section{Study cohort}

In the MRN group, from June 2018 to December 2018, a total of 53 patients were admitted, seven cases were excluded, and 46 cases were eligible for our research (Figure S1).

In the non-MRN group, from January 2016 to March 2018, a total of 205 patients were admitted, 55 cases were excluded, and 150 cases were considered eligible for our research (Figure S1).

The characteristics of the two groups are summarized in Table 2, which shows there was no significant difference between the two. There was also no significant difference in perioperative results between the two groups, as shown in Table 3. The kappa value of consistency evaluation of gastrointestinal surgeons and radiologists on the conformity of MRN images with anatomical structures was 0.72 , indicating that inter-observer agreement between the two was strong (Table 4).

\section{Postoperative urinary and sexual function}

Changes in total IPSS, IIEF5, ICIQ-FLUTS, and FSFI scores after surgery are shown in Table 5. In male patients, based on IPSS and IIEF5 scores at 6 months, postoperative urinary and sexual function in the MRN group were significantly better than in the non-MRN group $(\mathrm{P}<0.05)$. Postoperative urinary and sexual function at 6 months were significantly worse than preoperative urinary and sexual function of the two groups $(\mathrm{P}<0.05)$. In female patients, based on ICIQ-FLUTS and FSFI scores at 6 months, postoperative sexual function in the MRN group was significantly better than in the non-MRN group $(\mathrm{P}<0.05)$, and there was no significant difference in postoperative urinary function between the groups $(\mathrm{P}>0.05)$. There was also no significant difference between postoperative results and preoperative results of the MRN group $(\mathrm{P}>0.05)$.

\section{POV}

The POV of autonomic nerves intraoperatively is summarized in Table 6. The POV of NVB and PSN in the $M R N$ group were significantly higher than that of the nonMRN group $(\mathrm{P}<0.05)$, while the POV of SHP, HGN, PP, and the sympathetic nerve at the root of the IMA showed no statistical significance $(\mathrm{P}>0.05)$. A logistic regression analysis identified MRN as an independent factor that could increase the POV of NVB and PSN (Tables 7,8).

\section{MRN atlas of PANS}

The PANS shown in MRN images were compared with those shown intraoperatively (Figures 1-3), and continuous sagittal MRN images and the $3 \mathrm{D}$ model are shown in Figure 4 and Figure 5. The POV of the SHP, HGN, PP, PSN, NVB, and pudendal nerve in preoperative MRN were $61 \%, 93 \%, 65 \%$, 93\%, 61\%, and 93\%, respectively.

\section{Postoperative MRN}

The POV of the SHP, HGN, PP, PSN, NVB, and pudendal nerve in the postoperative MRN image were $50 \%, 89 \%$, $54 \%, 91 \%, 54 \%$, and $87 \%$, respectively, and compared with preoperative MRN results, there was no significant statistical difference, with the results shown in Table 9.

\section{Discussion}

To our knowledge, this is the first report of the application of MRN to PANS in patients with RC. Previous anatomy studies on PANS have mainly included autopsy and serial histology based on cadavers (15-17), and have been unable 
Table 2 Patient demographics and clinical characteristics

\begin{tabular}{|c|c|c|c|}
\hline Variable & MRN group $(n=46)$ & Non-MRN group $(n=150)$ & $P$ value \\
\hline Sex, n (\%) & & & 0.50 \\
\hline Male & 34 (73.91) & $120(80.00)$ & \\
\hline Female & $12(26.09)$ & $30(20.00)$ & \\
\hline Tumor distance from AV (cm), n (\%) & & & 0.84 \\
\hline$\leq 8$ & $24(52.17)$ & $83(55.33)$ & \\
\hline $8-12$ & $22(47.83)$ & $67(44.67)$ & \\
\hline cT, n (\%) & & & 0.36 \\
\hline 3 & $19(41.30)$ & $72(48.00)$ & \\
\hline $4 a$ & $2(4.35)$ & $16(10.67)$ & \\
\hline $\mathrm{cN}, \mathrm{n}(\%)$ & & & 0.74 \\
\hline 0 & 34 (73.91) & $102(68.00)$ & \\
\hline 1 & $9(19.57)$ & $37(24.67)$ & \\
\hline 2 & $3(6.52)$ & $11(7.33)$ & \\
\hline nCRT, n (\%) & $21(46.65)$ & $88(58.67)$ & 0.12 \\
\hline IPSS & $1.82 \pm 1.51$ & $1.94 \pm 1.32$ & 0.94 \\
\hline
\end{tabular}

MRN, magnetic resonance neuroimaging; BMI, body mass index; AV, anal verge; nCRT, neo-adjuvant chemoradiotherapy; IPSS, International Prostate Symptom Score; IIEF5, International Index of Erectile Function 5; FSFI, Female Sexual Function Index; ICIO-FLUTS, International Consultation on Incontinence Questionnaire Female Lower Urinary Tract Symptoms.

to generate a presentation of PANS in vivo and provide individualized guidance during clinical practice. Recent advances in MRN have seen its application to healthy volunteers (18) and patients with cervical cancer, but not to patients with RC. In our study, a personalized autonomic nerve atlas of RC patients was constructed to confirm the course and distribution of PANS and the location of the tumor so that individual anatomical variations could be identified. A personalized surgical plan of how to preserve PANS well was developed accordingly.

Previous intraoperative preservation of PANS mainly relied on the experience of surgeons and intraoperative visibility assessment, which could be easily affected by factors such as pelvic stenosis, rich adipose tissue, and intraoperative bleeding (6). When autonomic nerves are not visible during the operation, the results are usually unsatisfactory if mechanically and blindly preserved in a fixed direction in all patients. A survey on the POV of PANS during TME surgery among European and American senior doctors showed that the POV of SHP, HGN, PP, PSN, and NVB was $58 \%, 81 \%, 44 \%, 13 \%$, and $32 \%$, respectively. Our results demonstrated preoperative MRN could increase the POV of the NVB and PSN in the MRN group. Urogenital dysfunction is principally related to injury of the nerve fibers of PANS, which is usually irreversible (19), and great attention needs to be 
Table 3 Perioperative results

\begin{tabular}{|c|c|c|c|}
\hline Variable & MRN group $(n=46)$ & Non-MRN ( $n=150)$ & $P$ value \\
\hline LAR & $28(60.87)$ & $94(62.66)$ & \\
\hline uLAR & $15(32.61)$ & $44(29.33)$ & \\
\hline Parks & $0(0.00)$ & $1(0.67)$ & \\
\hline Diverting ileostomy, n (\%) & $10(21.74)$ & $36(24.00)$ & 0.75 \\
\hline Operative time (min) & $150.00 \pm 9.21$ & $153.61 \pm 9.11$ & 0.19 \\
\hline Blood loss (mL) & $33.70 \pm 17.43$ & $35.20 \pm 24.92$ & 0.65 \\
\hline Degree of TME, n (\%) & & & 0.92 \\
\hline Incomplete & $1(2.17)$ & $5(3.33)$ & \\
\hline Positive lower margin, n (\%) & $0(0.00)$ & $1(0.67)$ & 1.00 \\
\hline Positive CRM, n (\%) & $0(0.00)$ & $2(1.33)$ & 1.00 \\
\hline 30-day mortality, n (\%) & $0(0.00)$ & $0(0.00)$ & 1.00 \\
\hline
\end{tabular}

MRN, magnetic resonance neuroimaging; LAR, low anterior resection; uLAR, ultra-low anterior resection; ISR, intersphincteric resection; CRM, circumferential resection margin; TME, total mesorectal resection.

Table 4 Pelvic autonomic nerve location reference and identifiability

\begin{tabular}{|c|c|c|c|c|}
\hline Nerve & Main positional reference & Identifiability [\%] & $\begin{array}{l}\text { Level of agreement } \\
\text { (radiologists) [\%] }\end{array}$ & Level of agreement (surgeons) [\%] \\
\hline SHP & $\begin{array}{l}\text { Division at or just below the level of } \\
\text { sacral promontory }\end{array}$ & 28 [61] & $28[100]$ & 28 [100] \\
\hline HGN & $\begin{array}{l}\text { Course just medial to internal iliac } \\
\text { vessels }\end{array}$ & $43[93]$ & $43[100]$ & $43[100]$ \\
\hline PP & Lateral wall of pelvis & 30 [65] & $30[100]$ & $30[100]$ \\
\hline NVB & Anterolateral of pelvis & 28 [61] & $21[75]$ & 24 [86] \\
\hline Pudendal nerve & Branch of 2 nd, 3rd, 4th sacral nerve & $43[93]$ & $43[100]$ & $43[100]$ \\
\hline
\end{tabular}

SHP, superior hypogastric plexus; HGN, hypogastric nerve; PSN, pelvic splanchnic nerve; PP, pelvic plexus; NVB, neurovascular bundle.

paid to the preservation of the HGN, PSN, and PP due to their variation. Aurore et al. (20) revealed that an accessory HGN could be found in $25 \%$ of samples, with two main patterns observed. In $56 \%$ of samples thick robust nerve bundles were found, while in $44 \%$ a rough meshwork of fibers intermingling with blood vessels was seen. The PSN joined PP by crossing the medially situated blood vessels without obvious pattern, and the ventral branches of S1 could also be seen as a component of the PSN in $18 \%$ samples. Accordingly, it is important to identify the course 
Table 5 Postoperative urogenital function of MRN and non-MRN group

\begin{tabular}{lccc}
\hline Functional evaluation & After nCRT (before operation) & 6 months after operation & P value \\
\hline IPSS & & & $0.00^{\star *}$ \\
MRN group & $2.18 \pm 1.57$ & $3.24 \pm 1.23$ & $0.00^{\star *}$ \\
Non-MRN group & $2.28 \pm 1.32$ & $4.05 \pm 1.41$ & $0.00^{* *}$ \\
IIEF5 & & & $0.04^{*}$ \\
MRN group & $22.29 \pm 1.36$ & $20.56 \pm 1.94$ & $0.00^{* *}$ \\
Non-MRN group & $22.68 \pm 1.23$ & $19.78 \pm 1.88$ & 0.43 \\
ICIQ-FLUTS & & & 0.79 \\
MRN group & $4.17 \pm 1.64$ & $4.33 \pm 1.44$ & 0.06 \\
Non-MRN group & $3.97 \pm 1.61$ & $4.73 \pm 1.48$ & $0.02^{*}$ \\
FSFI & & & 0.08 \\
MRN group & $29.75 \pm 2.99$ & $27.67 \pm 2.50$ & $0.00^{\star *}$ \\
Non-MRN group & $29.80 \pm 2.55$ & $25.53 \pm 2.03$ & \\
\hline
\end{tabular}

${ }^{*}, \mathrm{P}<0.05$; ${ }^{*}, \mathrm{P}<0.01$. MRN, magnetic resonance neuroimaging; IPSS, International Prostate Symptom Score; IIEF5, International Index of Erectile Function 5; FSFI, Female Sexual Function Index; ICIO-FLUTS, International Consultation on Incontinence Questionnaire Female Lower Urinary Tract Symptoms.

Table 6 Prevalence of visibility intraoperatively

\begin{tabular}{lccc}
\hline Autonomic nerve & MRN group $(n=46)[\%]$ & Non-MRN group $(n=150)[\%]$ & $P$ value \\
\hline SHP & $30[65]$ & $91[61]$ & 0.58 \\
HGN & $41[89]$ & $114[76]$ & 0.06 \\
PP & $32[70]$ & $80[53]$ & 0.05 \\
PSN & $18[39]$ & $29[19]$ & $0.01^{\star \star}$ \\
NVB & $25[54]$ & $47[31]$ & $0.00^{\star \star}$ \\
Sympathetic trunk of IMA & $23[50]$ & $70[47 \%]$ & 0.69 \\
\hline
\end{tabular}

**, $\mathrm{P}<0.01$. MRN, magnetic resonance neuroimaging; SHP, superior hypogastric plexus; HGN, hypogastric nerve; PSN, pelvic splanchnic nerve; PP, pelvic plexus; NVB, neurovascular bundle.

and distribution of the HGN and PSN through MRN to avoid damage (21). Runkel et al. (22) developed a nerveoriented mesorectal excision (NOME) as a novel concept in RC surgery by which autonomic pelvic nerves serve as landmarks for a standardized navigation along fascial planes. Three vulnerable sites in laparoscopy TME + ANP such as the HGN, PSN, and PP could be clearly seen on our 3D MRN model. Based on the preoperative MRN images, we performed nerve-oriented laparoscopic TME individually so that the PANS could be preserved intact. As a result, our questionnaire-based results showed that postoperative urogenital function in the MRN group were better than that of the non-MRN group except for urinary function in female patients. This shows MRN can promote the preservation of PANS during nerve-oriented laparoscopic TME + ANP. However, in the case of a lot of intraoperative bleeding and severe abdominal and pelvic adhesion, the neuroprotection of laparoscopic TME will encounter great difficulties.

Previous evaluations of postoperative urinary and sexual function have been performed using questionnaire-based evaluations $(9,23)$, including the IPSS, IIEF5 and FSFI, and ICIQ-FLUTS. However, questionnaire-based evaluation is subjective and can be affected by the patient's emotions, 
Table 7 Variables of prevalence of visibility of PSN according to logistics regression

\begin{tabular}{|c|c|c|c|c|}
\hline Variable & \multicolumn{2}{|c|}{ Univariate } & \multicolumn{2}{|c|}{ Multivariate } \\
\hline Age (years) & $0.98(0.93-1.03)$ & 0.38 & - & - \\
\hline Sex & $1.83(0.87-3.87)$ & 0.11 & - & - \\
\hline MRN & $0.37(0.18-0.76)$ & $0.01^{\text {** }}$ & $0.40(0.19-0.82)$ & $0.01^{* *}$ \\
\hline Tumor distance from AV & $1.35(0.70-2.60)$ & 0.37 & - & - \\
\hline cT & $0.65(0.41-1.03)$ & $0.07^{*}$ & $0.73(0.27-2.00)$ & 0.54 \\
\hline $\mathrm{cN}$ & $1.02(0.60-1.73)$ & 0.95 & - & - \\
\hline nCRT & $0.56(0.29-1.08)$ & $0.09^{*}$ & $0.90(0.21-3.76)$ & 0.88 \\
\hline Blood loss (mL) & $0.99(0.97-1.01)$ & 0.33 & - & - \\
\hline
\end{tabular}

*, $\mathrm{P}<0.10 ;{ }^{* *}, \mathrm{P}<0.01$. MRN, magnetic resonance neuroimaging; BMI, body mass index; AV, anal verge; nCRT, neo-adjuvant chemoradiotherapy.

Table 8 Variables of prevalence of visibility of NVB according to logistics regression

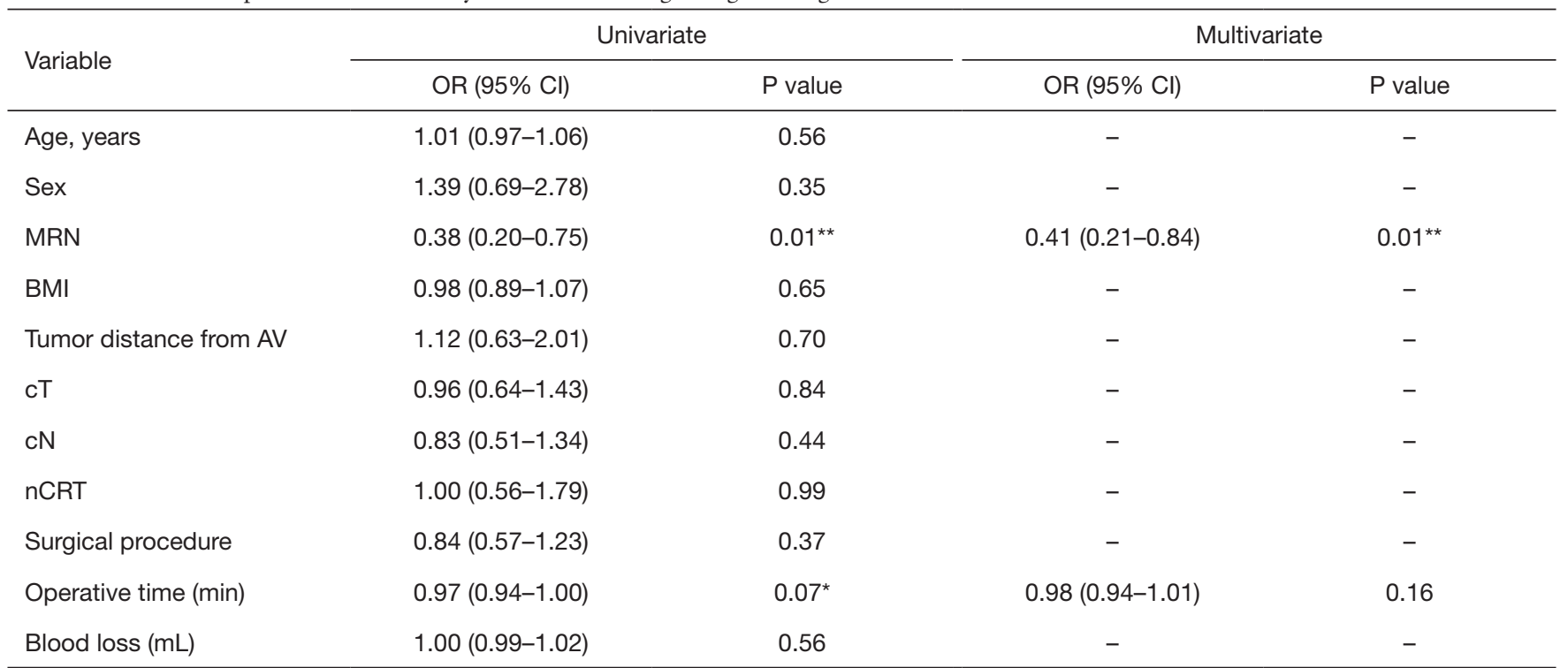

*, $\mathrm{P}<0.10 ;{ }^{*}, \mathrm{P}<0.01$. NVB, neurovascular bundle; MRN, magnetic resonance neuroimaging; BMI, body mass index; AV, anal verge; nCRT, neo-adjuvant chemoradiotherapy.

perceptions, and cognitive level. The combination of subjective and objective evaluation could better evaluate the postoperative urinary and sexual function, but to date, there have been a lack of objective indicators. In this study we explored whether postoperative MRN could be used as an objective indicator of evaluation because nerve damage showed on MRN images often lead to urinary and sexual dysfunction.

According to NCCN guidelines (24), we chose to perform postoperative MRI and MRN 6 months after surgery as 

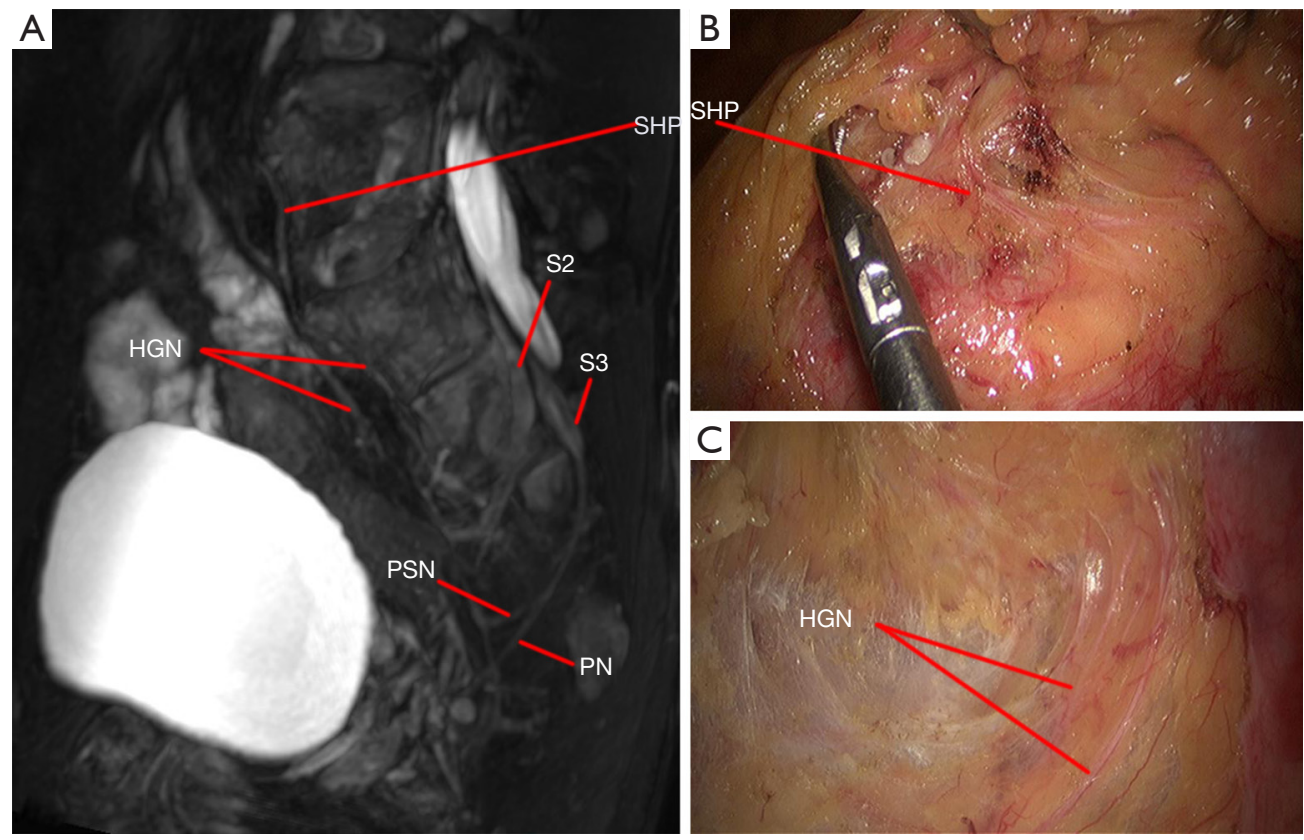

Figure 1 Preoperative MRN image and intraoperative SHP and HGN. (A) Sagittal MRN image, the SHP is visible, the HGN is separated at the sacral promontory. S2 and S3 can be seen to reach pelvic organs through the intervertebral foramen and send off two main branches. The PSN converged with the HGN to form the PP, and the other branch PN is separated; (B) the SHP is visible during surgery; (C) the $\mathrm{HGN}$ is visible when separating the posterior wall and lateral posterior wall, with three to five main branches moving towards the pelvic side wall. MRN, magnetic resonance neuroimaging; SHP, superior hypogastric plexus; HGN, hypogastric nerve; S2, second sacral nerve; S3, third sacral nerve; PSN, pelvic splanchnic nerve; PP, pelvic plexus; PN, pudendal nerve.

at that time, patients have usually recovered from surgical injury and postoperative tissue edema. Our results showed no significant difference in POV between preoperative and postoperative MRN, which meant that the neuromorphics were well preserved during the operation. This may be due to the use of 3D laparoscopy, experienced surgeons, and preoperative MRN (12). However, postoperative urinary and sexual function based on questionnaire-based evaluation 6 months after operation were worse than that preoperatively in the MRN group. The most likely reason for this is because postoperative MRN examination was mainly a morphological examination. Nerve ischemic injury, radiation injury, or drug-induced injury might result in a loss of function, but this does not affect the neuromorphics. Therefore, MRN examination, which is based on morphology, needs to be combined with questionnaire-based evaluation. Nevertheless, MRN may be used as one of the objective indicators to judge the recovery of postoperative sexual function and urinary function, as neural existence is the basis for function.

Furthermore, the MRN results could be used as the standard to perfect the operation. If the PANS failed to be displayed on postoperative MRN image while being clearly displayed on preoperative MRN image, the surgical video could be reviewed and the cause of nerve injury more accurately determined. Therefore, objective MRN results can be used to test the effect of autonomic nerve preservation during surgery, reflect on the deficiencies of the surgical process, and promote TME + ANP. According to our experience, MRN examination is required before surgery. An individualized surgical plan should be developed based on the MRN images and special attention should be paid to whether there are neurological mutations. During the operation, separation along the fascia gap between PHGNF and rectal propria fascia. The position with abundant autonomic nerves requires more delicate operation. Special attention should be paid to the course of the hypogastric nerve, pelvic splanchnic nerve and pelvic plexus when separating the posterior and lateral walls of the rectum, and to the protection of NVB when separating the anterior wall.

The main limitation of our study was that there were 

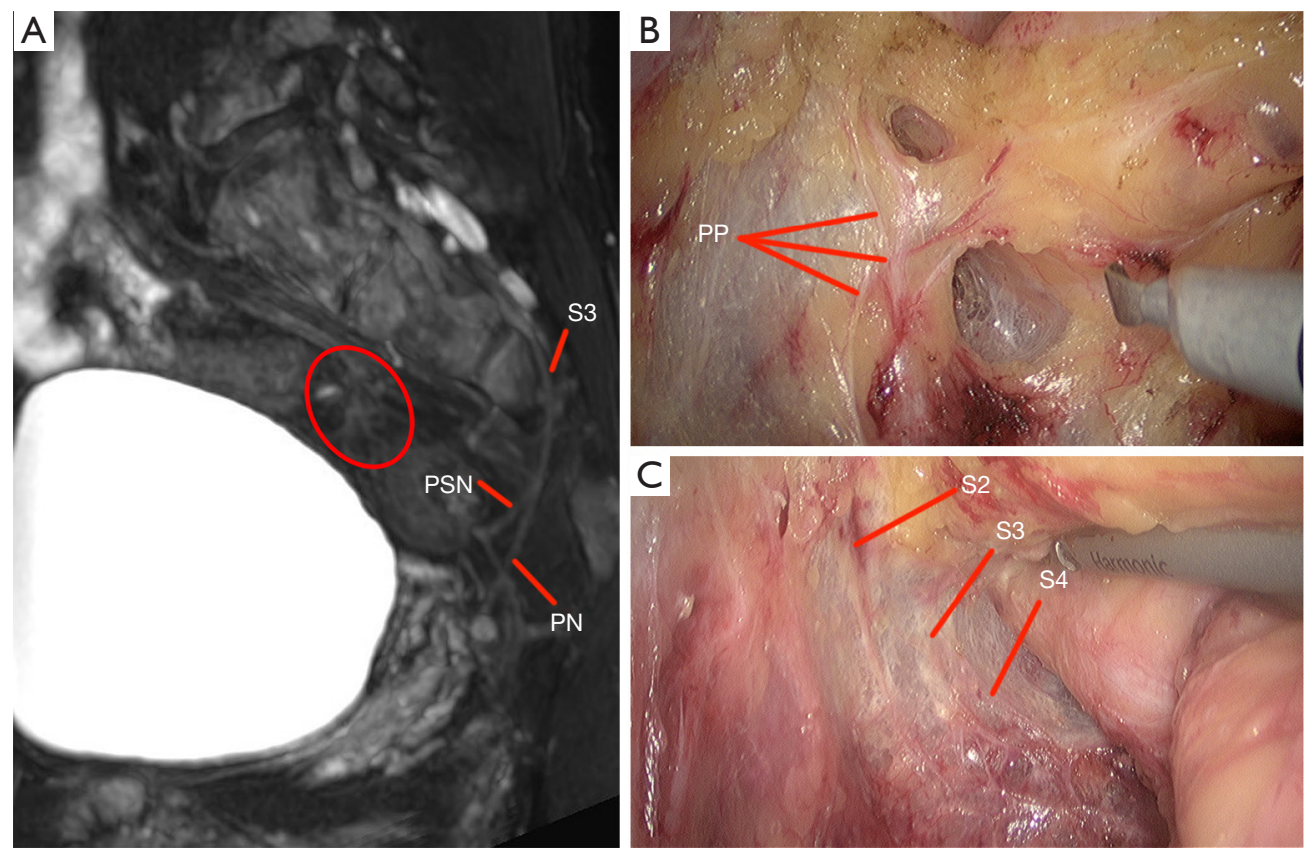

Figure 2 Preoperative MRN image and intraoperative PP and PSN. (A) Sagittal MRN image; the red circle area is the PP, which is distributed in a network. S3 sent out the PSN and PN; (B) the PP is visible on the pelvic side wall; (C) the PSN is seen when separating the posterolateral wall. MRN, magnetic resonance neuroimaging; PP, pelvic plexus; PSN, pelvic splanchnic nerve; S3, third sacral nerve; PN, pudendal nerve.
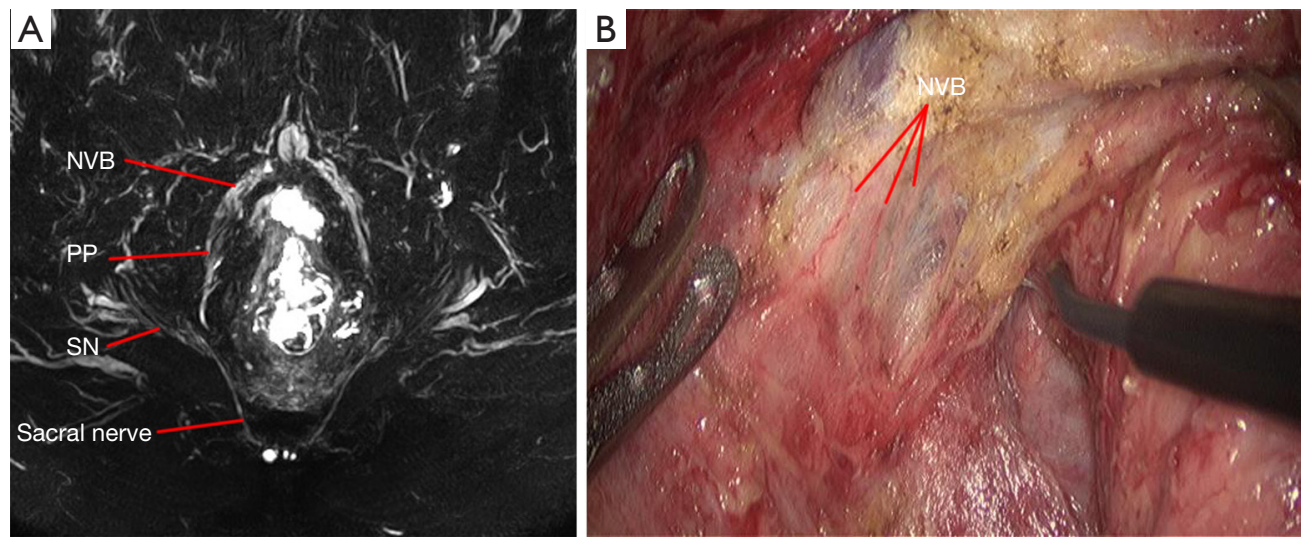

Figure 3 Preoperative MRN image and intraoperative NVB. (A) MRN image showing the sacral nerve and sciatic nerve. The PP is located on the lateral wall and the NVB is divided into several branches; (B) the NVB is seen when separating the anterior lateral wall. MRN, magnetic resonance neuroimaging; $\mathrm{SN}$, sciatic nerve; $\mathrm{PP}$, pelvic plexus; NVB, neurovascular bundle.

temporal differences and selection bias between the MRN group and the non-MRN group, including technological progress and device progress. The MRN group was enrolled in a non-blinded manner, which may have resulted in more refined surgery in this group. Further, the study was retrospective and involved a single institution. Long-term outcomes require further follow-up and analysis, ideally in a multiple-center, randomized control trial.

\section{Conclusions}

In the present study, we constructed a 3D presentation of PANS based on preoperative MRN which helped guide TME + ANP. This could promote the preservation of pelvic 

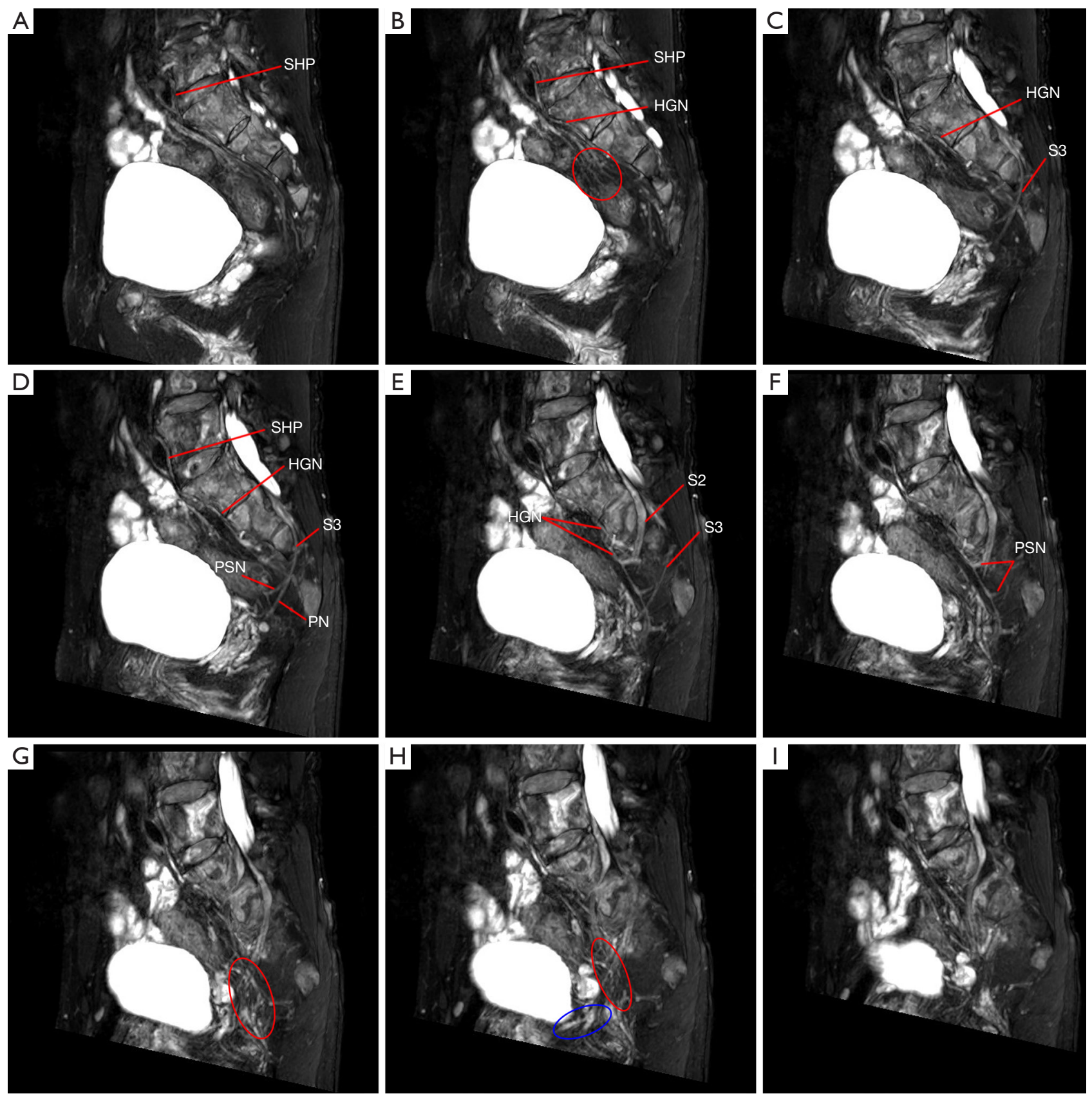

Figure 4 Sagittal MRN image. (A) The SHP is clearly visible; (B) the SHP sends out left and right HGN at the sacral promontory, and the red circle area is the PP innervated lateral wall of the pelvis, distributed in a network pattern; (C-F) S3 travels from the spinal cord through the foramen to the pelvic cavity. S3 sends out a branch PSN to converge with the HGN, while sending out another branch pudendal nerve; $(\mathrm{G}, \mathrm{H})$ the red circle area is the PP, and the blue circle area is the NVB; (I) pelvic autonomic nerve on the other sagittal plane. MRN, magnetic resonance neuroimaging; SHP, superior hypogastric plexus; HGN, hypogastric nerve; S2, second sacral nerve; S3, third sacral nerve; PSN, pelvic splanchnic nerve; PP, pelvic plexus; PN, pudendal nerve; NVB, neurovascular bundle. 


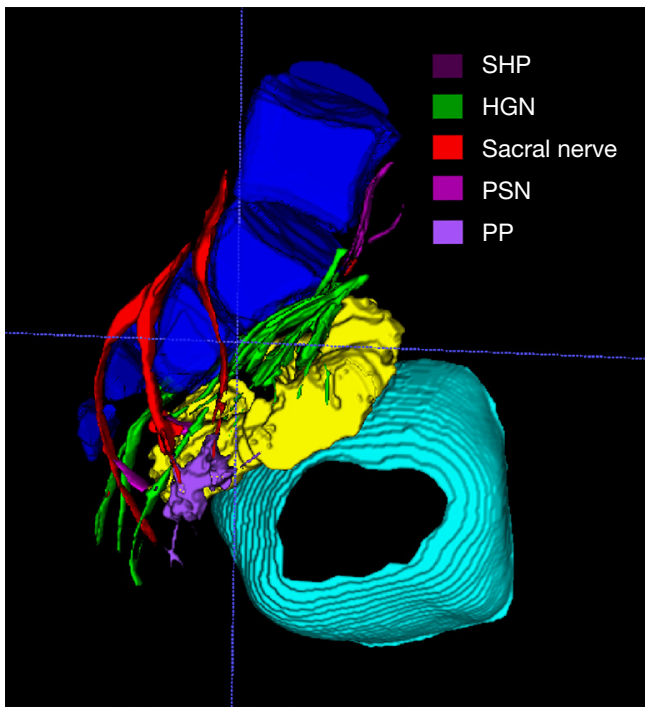

Figure $53 \mathrm{D}$ model based on MRN images. The following structures can be distinguished: bladder (indigo), rectum (yellow), sacrum (blue), SHP (deep red), HGN (green), sacral nerve (red), PSN (purple), PP (pink). MRN, magnetic resonance neuroimaging; SHP, superior hypogastric plexus; HGN, hypogastric nerve; PSN, pelvic splanchnic nerve; PP, pelvic plexus.

Table 9 Prevalence of visibility of postoperative and preoperative MRN

\begin{tabular}{lccc}
\hline $\begin{array}{l}\text { Autonomic } \\
\text { nerve }\end{array}$ & $\begin{array}{c}\text { Postoperative MRN } \\
(\mathrm{n}=46)[\%]\end{array}$ & $\begin{array}{c}\text { Preoperative MRN } \\
(\mathrm{n}=46)[\%]\end{array}$ & P value \\
\hline SHP & $23[50]$ & $28[61]$ & 0.29 \\
HGN & $41[89]$ & $43[93]$ & 0.71 \\
IHP (PP) & $25[54]$ & $30[65]$ & 0.40 \\
PSN & $42[91]$ & $43[93]$ & 1.00 \\
NVB & $25[54]$ & $28[61]$ & 0.67 \\
Pudendal nerve & $40[87]$ & $43[93]$ & 0.48 \\
\hline
\end{tabular}

MRN, magnetic resonance neuroimaging; SHP, superior hypogastric plexus; HGN, hypogastric nerve; PSN, pelvic splanchnic nerve; PP, pelvic plexus; NVB, neurovascular bundle.

autonomic nerves and reduce the postoperative urogenital dysfunction rate when ensuring a radical cure.

\section{Acknowledgments}

Funding: This study was supported by the Basic Research Plan of Guangzhou Science and Technology Plan Project (Item Number: 202002020082).

\section{Footnote}

Reporting Checklist: The authors have completed the STROBE reporting checklist. Available at https://dx.doi. org/10.21037/atm-21-5658

Data Sharing Statement: Available at https://dx.doi. org/10.21037/atm-21-5658

Conflicts of Interest: All authors have completed the ICMJE uniform disclosure form (available at https://dx.doi. org/10.21037/atm-21-5658). The authors have no conflicts of interest to declare.

Ethical Statement: The authors are accountable for all aspects of the work in ensuring that questions related to the accuracy or integrity of any part of the work are appropriately investigated and resolved. All procedures performed in this study involving human participants were in accordance with the Declaration of Helsinki (as revised in 2013). The study was approved by the clinical ethics committee of Sun Yat-sen Memorial Hospital, Sun Yat-sen University, Guangzhou, China (No. SYSEC-KYKS-2021-247) and informed consent was taken from all the patients.

Open Access Statement: This is an Open Access article distributed in accordance with the Creative Commons Attribution-NonCommercial-NoDerivs 4.0 International License (CC BY-NC-ND 4.0), which permits the noncommercial replication and distribution of the article with the strict proviso that no changes or edits are made and the original work is properly cited (including links to both the formal publication through the relevant DOI and the license). See: https://creativecommons.org/licenses/by-nc-nd/4.0/.

\section{References}

1. Hong YS, Kim SY, Lee JS, et al. Oxaliplatin-Based Adjuvant Chemotherapy for Rectal Cancer After Preoperative Chemoradiotherapy (ADORE): Long-Term Results of a Randomized Controlled Trial. J Clin Oncol 2019;37:3111-23.

2. Polanco PM, Mokdad AA, Zhu H, et al. Association of Adjuvant Chemotherapy With Overall Survival in Patients With Rectal Cancer and Pathologic Complete Response Following Neoadjuvant Chemotherapy and Resection. JAMA Oncol 2018;4:938-43. 
3. Heald RJ, Husband EM, Ryall RD. The mesorectum in rectal cancer surgery--the clue to pelvic recurrence? Br J Surg 1982;69:613-6.

4. Siegel RL, Miller KD, Jemal A. Cancer statistics, 2020. CA Cancer J Clin 2020;70:7-30.

5. Chen $W$, Zheng R, Baade PD, et al. Cancer statistics in China, 2015. CA Cancer J Clin 2016;66:115-32.

6. Duran E, Tanriseven M, Ersoz N, et al. Urinary and sexual dysfunction rates and risk factors following rectal cancer surgery. Int J Colorectal Dis 2015;30:1547-55.

7. Lange MM, van de Velde CJ. Urinary and sexual dysfunction after rectal cancer treatment. Nat Rev Urol 2011;8:51-7.

8. Celentano V, Cohen R, Warusavitarne J, et al. Sexual dysfunction following rectal cancer surgery. Int J Colorectal Dis 2017;32:1523-30.

9. Morelli L, Ceccarelli C, Di Franco G, et al. Sexual and urinary functions after robot-assisted versus pure laparoscopic total mesorectal excision for rectal cancer. Int J Colorectal Dis 2016;31:913-5.

10. Luca F, Craigg DK, Senthil M, et al. Sexual and urinary outcomes in robotic rectal surgery: review of the literature and technical considerations. Updates Surg 2018;70:415-21.

11. Li L, Bi Y, Wang L, et al. Identification and injury to the inferior hypogastric plexus in nerve-sparing radical hysterectomy. Sci Rep 2019;9:13260.

12. Bertrand MM, Macri F, Mazars R, et al. MRI-based 3D pelvic autonomous innervation: a first step towards imageguided pelvic surgery. Eur Radiol 2014;24:1989-97.

13. Li P, Liu P, Chen C, et al. The $3 \mathrm{D}$ reconstructions of female pelvic autonomic nerves and their related organs based on MRI: a first step towards neuronavigation during nervesparing radical hysterectomy. Eur Radiol 2018;28:4561-9.

14. Benson AB, Venook AP, Al-Hawary MM, et al. Rectal Cancer, Version 2.2018, NCCN Clinical Practice Guidelines in Oncology. J Natl Compr Canc Netw 2018;16:874-901.

15. Bertrand MM, Alsaid B, Droupy S, et al. Optimal plane for nerve sparing total mesorectal excision, immunohistological study and 3D reconstruction: an embryological study. Colorectal Dis 2013;15:1521-8.

16. Balaya V, Guimiot F, Uhl JF, et al. Three-Dimensional Modelization of the Female Human Inferior Hypogastric Plexus: Implications for Nerve-Sparing Radical
Hysterectomy. Gynecol Obstet Invest 2019;84:196-203.

17. Alsaid B, Bessede T, Karam I, et al. Coexistence of adrenergic and cholinergic nerves in the inferior hypogastric plexus: anatomical and immunohistochemical study with $3 \mathrm{D}$ reconstruction in human male fetus. J Anat 2009;214:645-54.

18. Yamashita R, Isoda H, Arizono S, et al. Selective visualization of pelvic splanchnic nerve and pelvic plexus using readout-segmented echo-planar diffusion-weighted magnetic resonance neurography: A preliminary study in healthy male volunteers. Eur J Radiol 2017;86:52-7.

19. Kim JY, Kim NK, Lee KY, et al. A comparative study of voiding and sexual function after total mesorectal excision with autonomic nerve preservation for rectal cancer: laparoscopic versus robotic surgery. Ann Surg Oncol 2012;19:2485-93.

20. Aurore V, Röthlisberger R, Boemke N, et al. Anatomy of the female pelvic nerves: a macroscopic study of the hypogastric plexus and their relations and variations. Journal of anatomy. 2020;237:487-94.

21. Kinugasa Y, Sugihara K. Topology of the Fascial Structures in Rectal Surgery: Complete Cancer Resection and the Importance of Avoiding Autonomic Nerve Injury. Seminars in Colon and Rectal Surgery 2010;21:95-101.

22. Runkel N, Reiser H. Nerve-oriented mesorectal excision (NOME): autonomic nerves as landmarks for laparoscopic rectal resection. Int J Colorectal Dis 2013;28:1367-75.

23. Attaallah W, Ertekin SC, Yegen C. Prospective study of sexual dysfunction after proctectomy for rectal cancer. Asian J Surg 2018;41:454-61.

24. Benson AB, Venook AP, Al-Hawary MM, et al. Colon Cancer, Version 2.2021, NCCN Clinical Practice Guidelines in Oncology. J Natl Compr Canc Netw 2021;19:329-59.

(English Language Editor: B. Draper)

Cite this article as: Zhong G, Yang B, Zhong J, Zhong Y, Zhi S, Shen J, Zhou S, Tan J, Huang J, Zhu J, Wang D, Han F. Magnetic resonance neuroimaging promotes the preservation of pelvic autonomic nerves in laparoscopic total mesorectal excision: a comparative study. Ann Transl Med 2021;9(24):1756. doi: 10.21037/atm-21-5658 
A total of 258 patients including 201 males and 57 females underwent laparoscopic TME+ANP between January 2016 and December 2018.

Excluded patients $(n=7)$ : abdominoperineal resection $(n=3)$ postoperative anastomotic leakage $(n=2)$ number of harvested lymph nodes $<12(\mathrm{n}=2)$

MRN group (June 2018-December 2018): MRN were performed in eligible patients before operation, including 39 males and 14 females $(n=53)$

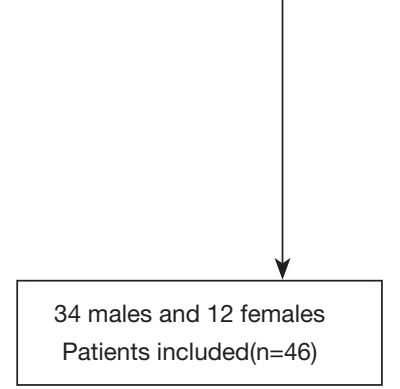

Excluded patients $(n=55)$ :

abdominoperineal resection $(n=7)$

postoperative anastomotic leakage $(n=11)$ number of harvested lymph nodes $<12(n=8)$ lateral dissection of pelvic lymph nodes $(n=2)$ conversion to open surgery $(n=2)$ urogenital dysfunction evaluated by forms $(n=25)$

Figure S1 The enrollment of MRN group and non-MRN group. MRN, magnetic resonance neuroimaging. 